\title{
A Case of Stroke and Blindness
}

\author{
Alicia Mattia, Michael Sharma, Michael Nicolle, J. Alexander Fraser, \\ Donald Lee, Robert Hammond
}

Can. J. Neurol. Sci. 2011; 40: 728-733

\section{Case Presentation: Dr. Mattia}

\section{History}

An 82-year-old woman presented to the emergency department with a one-day history of vomiting and vertigo. She did not have a headache, neck pain or history of recent trauma. She reported jaw pain two months prior to presentation, which had spontaneously resolved.

Her past medical history included type 2 diabetes mellitus, hypothyroidism, and hypercholesterolemia. She was taking metformin $500 \mathrm{mg}$ twice daily, levothyroxine $25 \mathrm{mcg}$ daily, simvastatin $40 \mathrm{mg}$ daily and acetylsalicylic acid (ASA) $81 \mathrm{mg}$ daily.

She was a lifelong non-smoker.

\section{Physical Examination}

On examination, she had right-beating nystagmus on right gaze. Power was normal with the exception of grade $4 / 5$ strength in her left deltoid. There was finger-to-nose dysmetria on the left side, dysdiadochokinesia with the left hand, and an unsteady gait. The remainder of her physical exam was unremarkable.

\section{NeURORADIOLOGY: DR. LEE}

Magnetic resonance imaging (MRI) demonstrated bilateral posterior inferior cerebellar artery distribution infarcts (larger on the left) with diffusion restriction, as well as two small right pontine perforator infarcts. The $3 \mathrm{D}$ phase contrast magnetic resonance angiography (MRA) showed absence of identifiable flow in both distal vertebral arteries, and T2 MRI showed an absence of intradural flow void in the left vertebral artery (Figure 1). Digital subtraction angiography (DSA) was not performed, considering the patient's age and the presence of a stroke. Collateral circulation to the basilar and vertebral arteries was presumed to be from both posterior communicating arteries, which were of reasonable calibre.

\section{Case Presentation: Dr. Mattia}

The patient was admitted to hospital. Aspirin was discontinued, and she was started on clopidogrel (75mg daily). Investigations did not reveal a cardiac source for her stroke.
Computed tomographic angiography (CTA) was not performed on the patient (possibly because she was diabetic, and vascular imaging was obtained with MRA). She was discharged to a rehabilitation facility.

Approximately three weeks later, while at the rehabilitation facility, she awoke completely blind. She had no pain, and no preceding ocular or neurological symptoms. She returned to the emergency department.

On examination her blood pressure was 123/60 $\mathrm{mmHg}$. She was afebrile and had no cardiac murmurs or carotid bruits. She had no light perception in either eye. Pupils were each $5 \mathrm{~mm}$, with absent direct and consensual reflexes. The optic discs were pale with blurred margins, and box-car segmentation was present in the retinal arterioles. The remainder of the neurological exam was unremarkable.

\section{DiscuSSANT: Dr. SHARMA}

We have an 82-year-old woman who has a posterior circulation infarct. The incidence of stroke is low before the age of 55, at which point it begins to rise exponentially, doubling for each subsequent decade. In women, stroke incidence is higher than myocardial infarction (MI) at older ages. In this instance there are multiple risk factors, which, coupled with age, increase her probability of large vessel disease, small vessel disease and atrial fibrillation. The frequency of involvement of the posterior circulation roughly follows the relative proportions of cerebral blood flow to this bed (20\% compared to $80 \%$ involving the anterior circulation). Large stroke data banks show that there is a slight excess of cardioembolic causes in posterior circulation stroke, though this is hampered by our ability to reliably assign etiologies.

Prior to this event, the patient was on ASA, presumably for primary prevention. In women there is some evidence to support the use of ASA for primary prevention of stroke though it does not appear to protect against ischemic heart disease in women. The reverse is true for men.

The patient has arrived outside any reasonable treatment window for thrombolysis and efforts should be directed at making an etiologic diagnosis. Preventive therapy is targeted against the underlying etiology, but frequently the treating

From the Departments of Clinical Neurological Sciences (AM, MN, JAF, DL, RH), Ophthalmology (JAF), Diagnostic Imaging (DL) and Pathology (RH), London Health Sciences

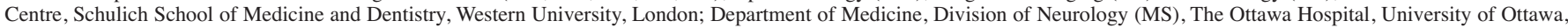
Ottawa, Ontario, Canada.

Received December 19, 2012. Final Revisions Submitted April 8, 2013.

Reprint requests to: Robert Hammond, Department of Pathology, London Health Sciences Centre, 339 Windermere Rd, London, Ontario, N6A 5A5, Canada.

Email: robert.hammond@lhsc.on.ca 

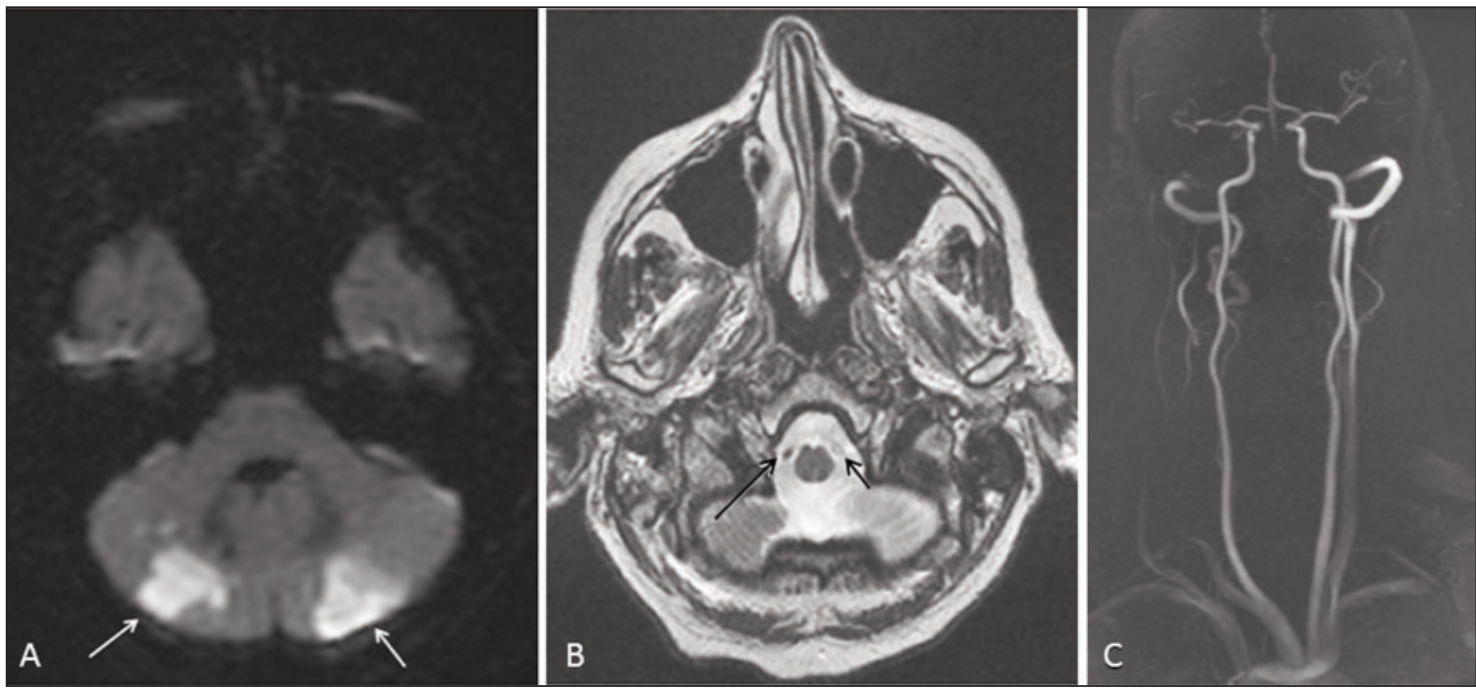

Figure 1: A) Magnetic resonance imaging (MRI) demonstrating bilateral posterior inferior cerebellar artery distribution infarcts (larger on the left) with diffusion restriction. B) T2 MRI demonstrating an absence of an intradural flow void in the left vertebral artery (short arrow) with a flow void evident on the right (long arrow). C) Magnetic resonance angiography (MRA) demonstrating markedly reduced or absent flow in both vertebral arteries.

physician is forced to make choices of antithrombotic therapy prior to the completion of the investigations. The substitution of clopidogrel for ASA is commonly done in our stroke prevention clinic (at the Ottawa Hospital), as the pivotal trial comparing these strategies suggested a 9\% relative risk reduction for clopidrogel over ASA for the endpoint of any vascular outcome in individuals with a broad range of vascular conditions. ${ }^{1}$ It should be acknowledged that there is no direct trial evidence to suggest that the use of clopidogrel (or any other antiplatelet agent) in someone who has a stroke while on ASA will be effective.

The initial presentation is compatible with posterior inferior cerebellar artery (PICA) territory infarcts with motor involvement reflecting the ischemia in the basis pontis. Holter monitor and echocardiogram did not provide a justification for anticoagulation. It should be noted that the prevalence of atrial fibrillation in the stroke population over the age of 80 exceeds $30 \%{ }^{2}$ with a substantial proportion being paroxysmal and not diagnosed at the time of presentation. The best duration for monitoring is not known, but emerging evidence suggests that many episodes of paroxysmal atrial fibrillation are asymptomatic and may require monitoring far longer than 48 hours to capture. It is my practice to use a two-week loop monitor in patients where there is a high index of suspicion and when the initial Holter monitor is negative.

The most significant finding on the initial MRI is the status of the vertebral arteries. Both show a severe attenuation or absence of flow in the distal portions. Magnetic resonance angiography may have difficulty distinguishing complete occlusion from low flow, and in our hands CTA is more sensitive for this purpose. While the most common cause of large vessel disease is atherosclerosis, selective involvement of both arteries is distinctly unusual. This pattern can be seen with dissection (usually with trauma) or with giant cell arteritis (GCA). Our group has observed the involvement of the vertebral arteries and the terminal internal carotid artery (ICA) in a number of cases of GCA. ${ }^{3,4}$ We consider this pattern to be strongly suggestive of the diagnosis. Thickening of the arterial wall in a smooth fashion is also more suggestive of GCA than atherosclerosis, which tends to look irregular and discontinuous. We have no history of trauma or neck pain but there is a history of jaw pain, which had resolved. Jaw claudication can be seen in GCA, though, to my knowledge, the resolution of this symptom is unusual. In addition, stroke is a described but rare presenting symptom of GCA. I would look for historical and laboratory support of this diagnosis by asking about headache, fever, anorexia and weight loss. In addition she should have measurement of complete blood count (CBC), erythrocyte sedimentation rate (ESR) and Creactive protein (CRP). The $\mathrm{CBC}$ might show anemia while an elevation of the ESR $>50 \mathrm{~mm} / \mathrm{hr}$ is strongly suggestive of GCA. Combining ESR with CRP increases the diagnostic sensitivity of these inflammatory markers.

Simultaneous arteritic anterior ischemic optic neuropathy (AION) is rare and blindness in this setting is more likely to be the result of bilateral occipital infarcts. The absence of pupillary reflexes seems to favor the optic nerves as the site of involvement. Physical examination and repeat imaging of the brain and vessels should help sort out the process.

\section{Case Presentation: Dr. Mattia}

Initial blood work revealed a normal CBC, normal serum electrolytes and creatinine. She had a low-density lipoprotein (LDL) of $1.2 \mathrm{mmol} / \mathrm{L}$, a fasting glucose of $8.4 \mathrm{mmol} / \mathrm{L}$. The patient's ESR was elevated at $83 \mathrm{~mm} / \mathrm{hr}$ and CRP was also elevated at $24.2 \mathrm{mg} / \mathrm{L}$.

A repeat echocardiogram and Holter monitor did not reveal any significant abnormalities. 

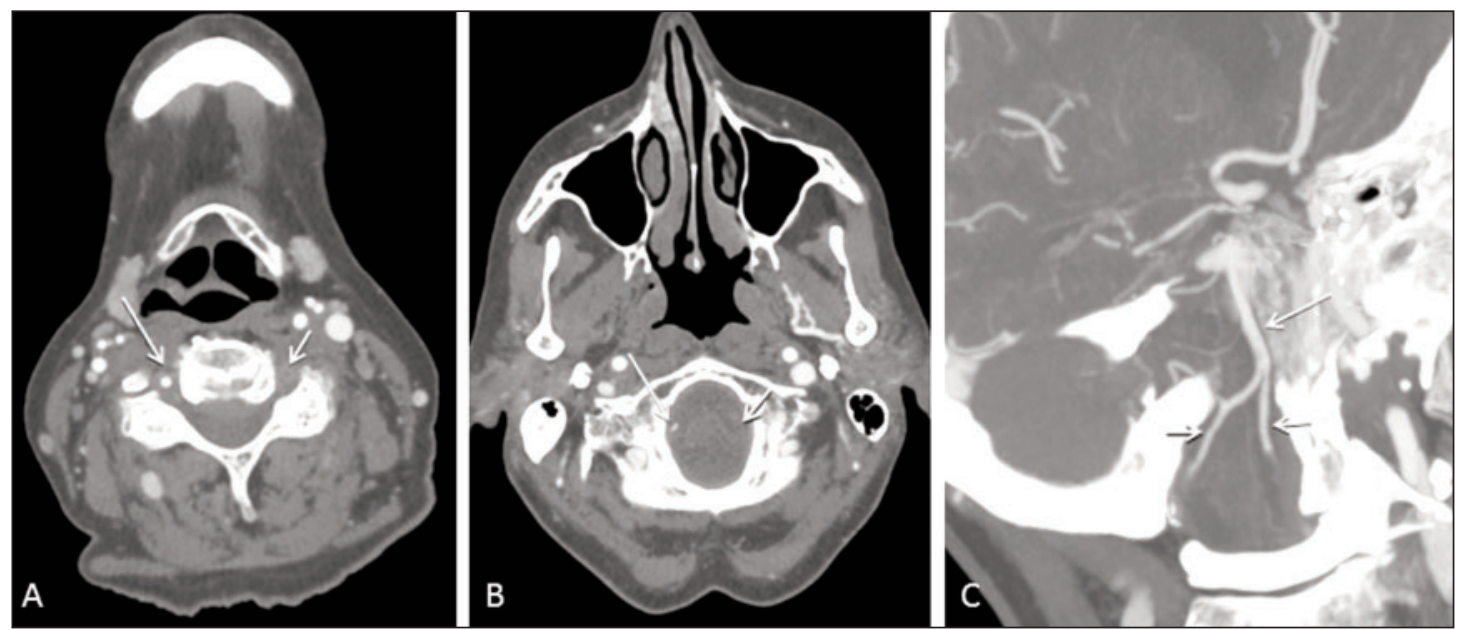

Figure 2: A, B) Computed tomography angiography (CTA) demonstrating patency with stenosis of the right vertebral artery in the neck (long arrows) and occlusion of the left vertebral artery (short arrows). C) Collateral flow appears to reconstitute the basilar (long arrow) and distal intradural vertebral arteries (short arrows) but with apparent occlusion of both proximal V4 segments.

\section{NeURORADiology: Dr. LeE}

Computed tomographic angiography done on her second presentation to hospital (Figure 2) showed patency of the right vertebral artery in the neck, with stenosis of the artery at $\mathrm{C} 7$ and almost complete loss of lumen at $\mathrm{C} 3$ upwards. There appeared to be collateral flow reconstituting the intradural right vertebral artery. The left vertebral artery was completely occluded in the neck, and the proximal V4 segment was also occluded, though the distal left V4 was patent. Apart from fenestration of the lower artery, no luminal changes of the basilar artery were seen. Both the left and right PICA, larger on the right, were seen on the CTA. There were no occipital infarcts.

\section{Neuro-Ophthalmology: Dr. Fraser}

Fundus photographs (Figure 3) of the right eye show "pallid" edema, two wedge-shaped patches of white retinal edema between the optic disc and the macula, and an old chorioretinal scar. The left fundus also has pallid edema and a wedge-shaped patch of retinal edema between the optic disc and the macula.

Intravenous retinal fluorescein angiography (Figure 4) was performed using sodium fluorescein dye, with photographs of the right eye taken under cobalt blue lighting to observe the retinal circulation at different time points. In a normal eye, fluorescein appears in the choroid as a diffuse "flush" approximately ten seconds after its injection into an antecubital vein. By 10-12 seconds, retinal arteries fill, followed by retinal vein filling at approximately the 15 second mark. The late venous stage occurs after $18-20$ seconds. ${ }^{5}$

In this patient, not only is there significant delay in choroid filling (19.25 seconds), but the choroid also fills in a patchy manner, suggesting areas of choroidal hypoperfusion and perhaps ischemia. There is also a cilioretinal artery that appears truncated and leaks dye, indicating a possible infarct in the
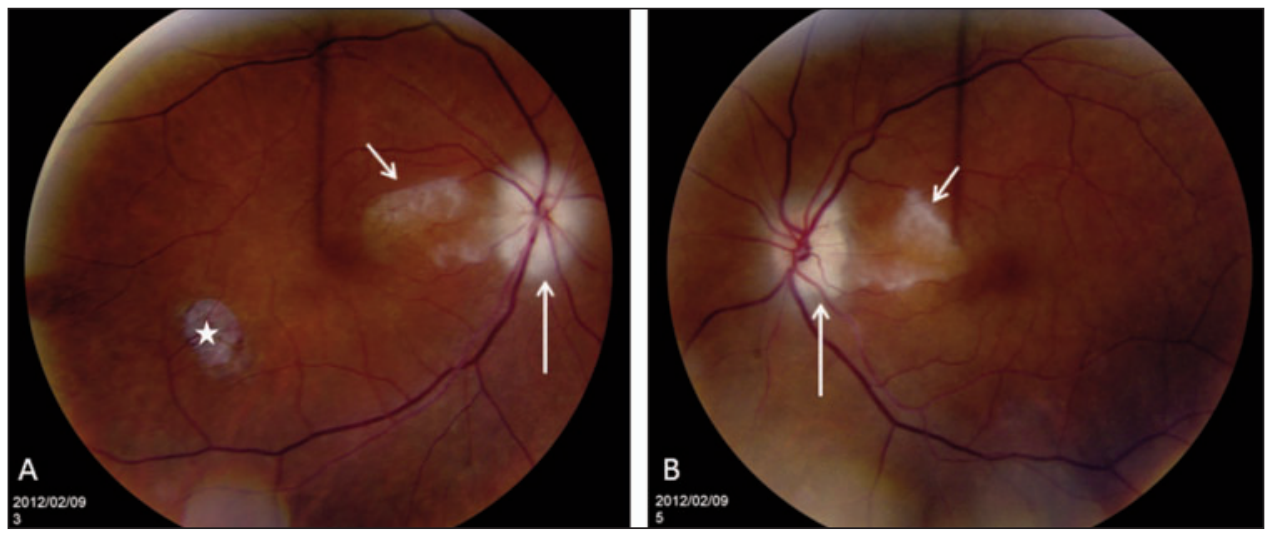

Figure 3: Photographs of the right $(A)$ and left $(B)$ ocular fundi, showing diffuse "pallid" edema of the optic nerves (long arrows) and retinal edema from cilioretinal artery occlusions (short arrows). (An old and unrelated incidental chorioretinal scar is also evident in the right eye (asterisk)). 

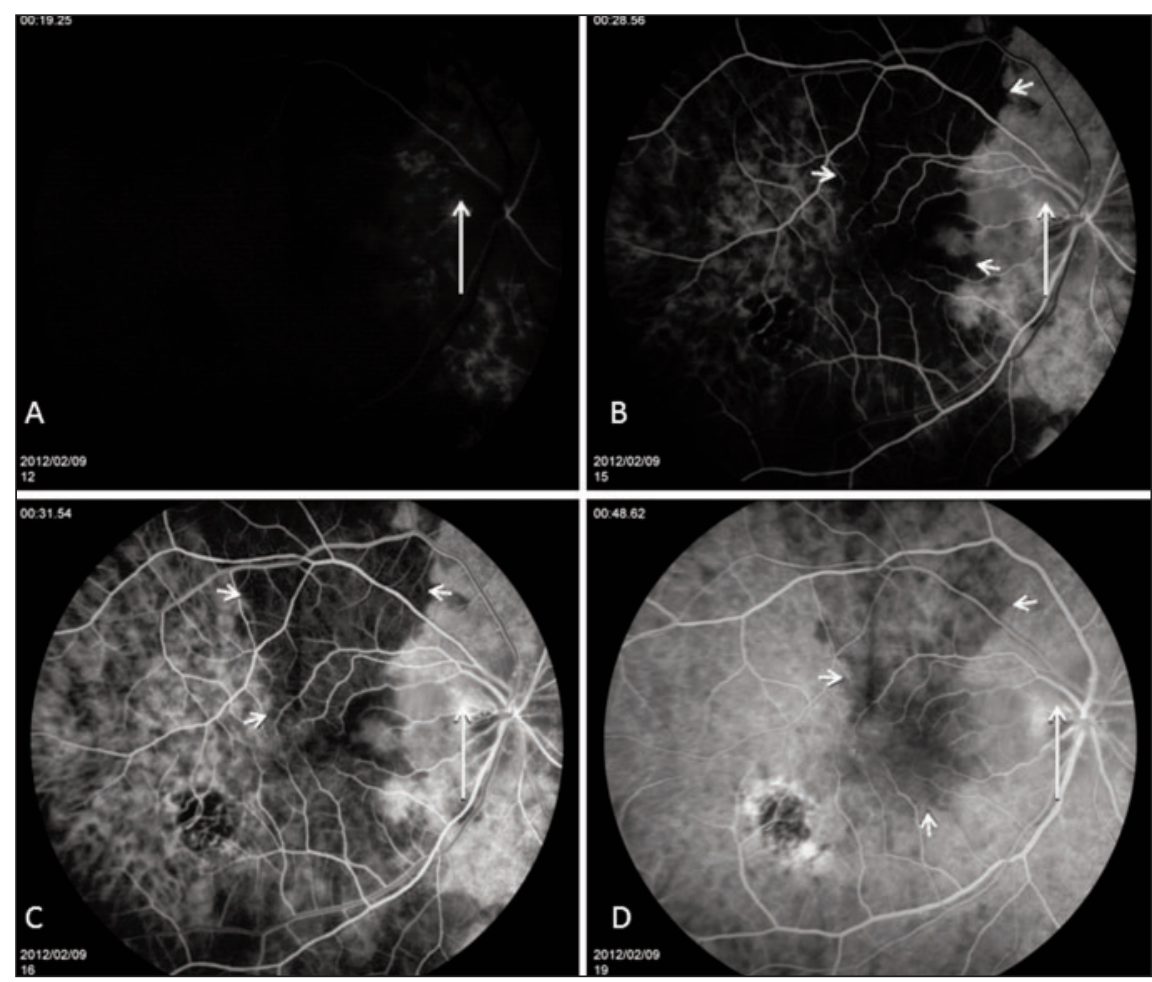

Figure 4: Intravenous retinal fluorescein angiography (IVFA) in the right eye, showing retinas at $19.25 \mathrm{~s}(\mathrm{~A}), 28.56 \mathrm{~s}(\mathrm{~B}), 31.54 \mathrm{~s}(\mathrm{C})$, and $48.62 s(D)$ after injection. Significantly delayed choroidal filling time is evident (19.25s; normal: 10s), with patchy choroidal hypoperfusion ensuing thereafter (short arrowheads), indicative of widespread or multifocal ischemia in the territory of the posterior ciliary artery. Central retinal artery filling time is also delayed $(19.25 \mathrm{~s}$; normal 10-12s). A cilioretinal artery (long arrows) appears truncated and leaks, indicating occlusion with distal retinal ischemia. Later phases of the IVFA (not shown) confirm the funduscopic impression of optic disc edema. Identical findings were evident in the left eye.

territory of the cilioretinal artery in the right eye (which corresponds to the wedge of retinal edema on fundus photographs). Similar angiographic findings were also evident in the left eye (photographs not shown).

\section{Discussant: Dr. Sharma}

The vascular imaging demonstrates significant involvement of both vertebral arteries with sparing of the intradural segment - a pattern usually seen with GCA. The ophthalmic examination suggests ischemic involvement of both optic nerves and the choroidal vessels. Again, simultaneous AION in the setting of GCA is rare and I wonder if it was not sequential with the first event not noticed by the patient. The ESR and CRP are significantly elevated suggesting an inflammatory process. I think the diagnosis is GCA but there are other conditions that can involve large and medium sized vessels. Takayasu's arteritis involves the great vessels at the level of the aortic arch but is not associated with optic nerve ischemia and tends to occur in younger individuals. Polyarteritis nodosa tends to be asymmetric and focal. Neither of these alternatives fits with the clinical and radiologic pattern. Temporal artery biopsy can confirm the diagnosis, and we usually do bilateral biopsies to avoid false negatives due to the segmental nature of the process. While I do not think the diagnosis is in doubt, pathologic confirmation of the diagnosis often provides the physician and patient with the confidence to carry on with long-term steroids when significant side effects occur.

Due to the involvement of the brain and visual loss, treatment should be started intravenously with subsequent daily oral therapy. The ESR and CRP are good markers to follow particularly while tapering the steroids. Glucocorticoids will cause an elevation in her blood sugar and I would suggest involving endocrinology for management of her diabetes and mitigation of the risk of osteoporosis associated with long-term steroid therapy. It should be noted that stroke may occur even after the initiation of steroid therapy ${ }^{4}$ and in rare instances other forms of immunosuppression have been employed.

\section{Neuro-Pathology: Dr. Hammond}

A temporal artery branch biopsy revealed typical changes of giant cell arteritis. The vessel wall was markedly thickened and the lumen severely narrowed (Figure 5). The internal elastic lamina was fragmented. Lymphohistiocytic inflammatory infiltrates with focal giant cell formation (Figure 5) were abundant, the extent of which is best appreciated with immunohistochemical preparations for lymphocytic (CD45) and histiocytic (CD68) markers (Figure 5).

\section{Topic Review: Dr. MatTia}

\section{Epidemiology}

Giant cell arteritis is a vasculitic disease that has an average annual incidence of 6.9-32.8 per 100,000 people aged 50 and older. Rates have varied between geographic regions, with a higher incidence typically found in more northern countries. However, in almost all regions, the rates of GCA in women have exceeded those in men, at a ratio of $3: 2 .^{6}$ 


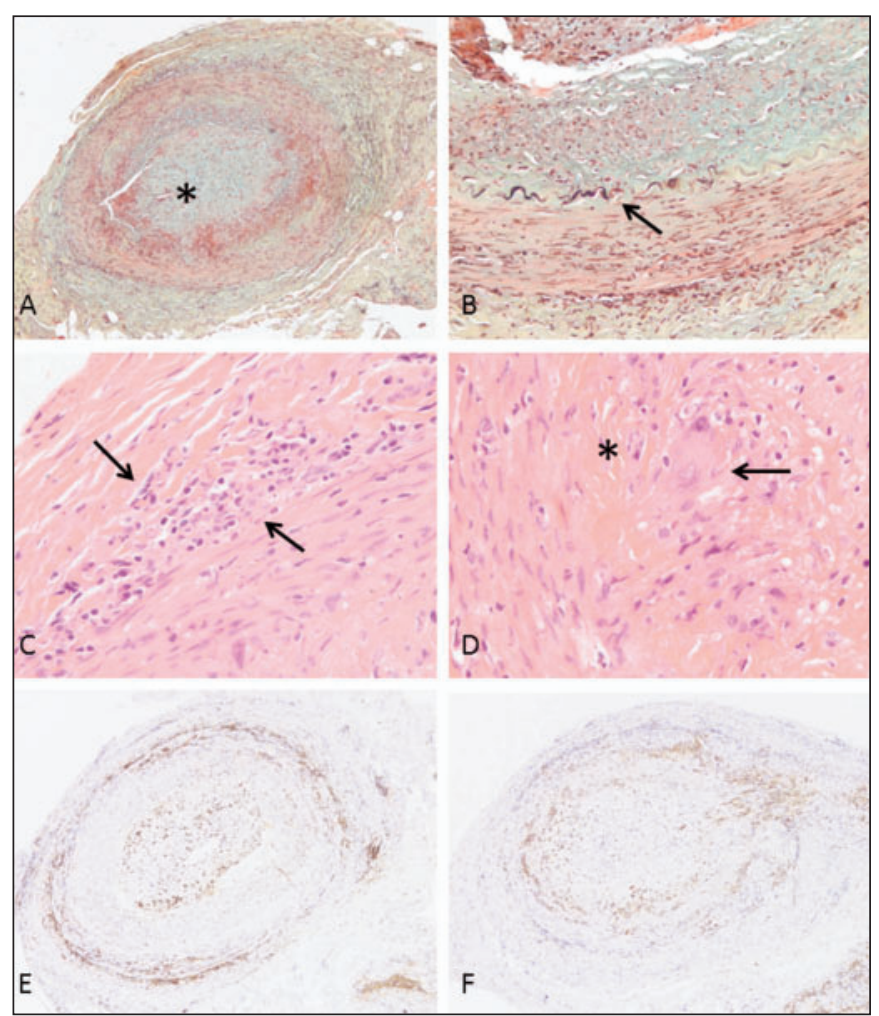

Figure 5: A) Marked mural thickening with near occlusion of lumen (small space to the left of asterisk), Movat's pentachrome stain (image width $=2 \mathrm{~mm})$. B) The internal elastic lamina (arrow) is fragmented and discontinuous, Movat's pentachrome stain (image width $=0.5 \mathrm{~mm}$ ). C) Lymphohistiocytic mural infiltrate (arrows), Hematoxylin and Eosin (image width $=0.25 \mathrm{~mm}$ ). D) Histiocyte giant cell formation (arrow). Internal elastic membrane remnant (asterisk) with media to the left and intima to the right, Hematoxylin and Eosin (image width $=0.25 \mathrm{~mm}$ ). E) Circumferential and transmural lymphocytic infiltrates, anti-CD45 immunohistochemistry (image width $=2 \mathrm{~mm}$ ). F) Circumferential and transmural histiocytic infiltrates, anti-CD68 immunohistochemistry (image width $=2 \mathrm{~mm})$.

\section{Clinical presentation}

Patients usually present with headache, joint pain, ocular symptoms, scalp tenderness, jaw claudication and/or constitutional symptoms. ${ }^{7}$ Headache is a presenting symptom in one third of GCA cases, but is eventually present in $72-90 \%$ of affected patients. Joint and back pain is often due to concurrent polymyalgia rheumatic (PMR), which is an inflammatory disorder present in over half $(\sim 58 \%)$ of patients with GCA. ${ }^{8}$

Ocular manifestations of the disease are present in up to $70 \%$ of patients, and can include transient vision loss (amaurosis fugax), diplopia, and permanent vision loss. Arteritic AION accounts for $78-99 \%$ of vision loss, with other etiologies (eg. central retinal artery occlusion, posterior ischemic optic neuritis and cortical blindness) occurring at a much lower frequency. 8,9

Some extra-ocular, neurologic manifestations of GCA include peripheral neuropathies, vertigo, neuropsychiatric disease, hemitongue numbness, and rarely acute myelopathy. ${ }^{10,11}$
Giant cell arteritis can cause large-vessel vasculitis, resulting in aortic aneurysm or dissection, subclavian or femoral claudication, and/or large artery ischemia. ${ }^{12,13}$ Although stroke is a rare presentation of GCA, arteritic involvement of the vertebrobasilar system may cause transient ischemic attack (TIA) or stroke in up to 4-7\% of patients with GCA. Compared to other patients with stroke, patients with GCA are at increased risk for ischemia in the vertebrobasilar system. ${ }^{3,8,10}$

The disease typically affects blood vessels that contain a significant amount of elastic tissue. Common sites of involvement include superficial temporal, ophthalmic, vertebral and posterior ciliary arteries. ${ }^{11}$

\section{Diagnosis}

Diagnosis is usually made using the 1990 Criteria for the Classification of Giant Cell (Temporal) Arteritis, which was published by the America College of Rheumatology (ACR). If there are at least three out of five positive criteria, the sensitivity and specificity for GCA are $93.5 \%$ and $91.2 \%$ respectively (Table 1). ${ }^{14}$

Table 1: The American College of Rheumatology 1990 criteria for the classification of giant cell arteritis ${ }^{14}$

1. Age of onset greater than 50 years

2. Onset of new headache

3. Temporal artery abnormality (tenderness or decreased pulsation)

4. Elevated erythrocyte sedimentation rate, defined as $50 \mathrm{~mm} / \mathrm{h}$ using the Westergren method

5. Abnormal arterial biopsy - showing vasculitis characterized by mononuclear cell infiltration or granulomatous inflammation

Temporal artery biopsy is important for establishing the diagnosis of GCA, and ideally should be done within a week of starting treatment with corticosteroids. ${ }^{7}$ In certain scenarios, consideration should be made of whether or not the results of a biopsy will change management. ${ }^{15}$

In cases where stroke or other large vessel disease is the first presentation of suspected GCA, various imaging modalities to assess the vasculature, such as MRA, CTA, angiography ${ }^{12}$ and even ultrasound ${ }^{16}$ can be useful diagnostic tools. ${ }^{3,4}$

\section{Management}

The usual treatment for GCA involves high-dose glucocorticoid administration. If the patient's vision is affected, intravenous methylprednisone $(1000 \mathrm{mg} /$ day $)$ is often given for three days, before switching to oral prednisone at $1-2 \mathrm{mg} / \mathrm{kg} / \mathrm{day}$. This dose is maintained for several weeks, and can be slowly 
tapered if inflammatory markers are returning to normal levels and symptoms are abating. If, during the taper, there is evidence of active disease the dose of steroids should be evaluated, and possibly increased. ${ }^{3}$ Relapses are frequent, but steroids can usually be discontinued within one to two years. ${ }^{6}$

\section{Conclusion}

When this patient initially presented to hospital with vertebral artery disease and cerebellar and pontine strokes, it was likely an unrecognized first manifestation of GCA. Stroke is a rare presentation of GCA. In a large case series, $0.15 \%$ of patients with first-ever stroke were diagnosed with this vasculitic disease. ${ }^{17}$

Following a full three-day course of intravenous methylprednisone, the patient was discharged home, but had no improvement in vision. Her inflammatory markers are followed at regular visits, and she has been tolerating a reduction in oral steroids.

\section{REFERENCES}

1. CAPRIE Steering Committee. A randomised, blinded, trial of clopidogrel versus aspirin in patients at risk of ischaemic events (CAPRIE). CAPRIE steering committee. Lancet. 1996;348 (9038):1329-39.

2. Marini C, De Santis F, Sacco S, et al. Contribution of atrial fibrillation to incidence and outcome of ischemic stroke: Results from a population-based study. Stroke. 2005;36(6):1115-19.

3. Zwicker J, Atkins EJ, Lum C, et al. An atypical presentation of giant cell arteritis. CMAJ. 2011;183(5):E301-5.

4. Tang V, Fantaneanu T, Chakraborty S, et al. Letter to the editor. Intracranial non-occlusive thrombus and multiple strokes in giant cell arteritis. Can J Neurol Sci. 2012;39(1):116-17.

5. Siatkowski RM, Gass JD, Glaser JS, et al. Fluorescein angiography in the diagnosis of giant cell arteritis. Am J Ophthalmol. 1993; 115(1):57-63

6. Hunder GG. Epidemiology of giant-cell arteritis. Cleve Clin J Med. 2002;69 Suppl 2:SII79-82.

7. Carroll SC, Gaskin BJ, Danesh-Meyer HV. Giant cell arteritis. Clin Experiment Ophthalmol. 2006;34(2):159-73.

8. Caselli RJ, Hunder GG. Neurologic complications of giant cell (temporal) arteritis. Semin Neurol. 1994;14(4):349-53

9. Chew SS, Kerr NM, Danesh-Meyer HV. Giant cell arteritis. J Clin Neurosci. 2009;16(10):1263-8.

10. Caselli RJ, Hunder GG, Whisnant JP. Neurologic disease in biopsyproven giant cell (temporal) arteritis. Neurology. 1988;38(3): 352-9.

11. Reich KA, Giansiracusa DF, Strongwater SL. Neurologic manifestations of giant cell arteritis. Am J Med. 1990;89(1):67-72.

12. Brack A, Martinez-Taboada V, Stanson A, et al. Disease pattern in cranial and large-vessel giant cell arteritis. Arthritis Rheum. 1999;42(2):311-17.

13. Evans J, Hunder GG. The implications of recognizing large-vessel involvement in elderly patients with giant cell arteritis. Curr Opin Rheumatol. 1997;9(1):37-40.

14. Hunder GG, Bloch DA, Michel BA, et al. The American College of Rheumatology 1990 criteria for the classification of giant cell arteritis. Arthritis Rheum. 1990;33(8):1122-8.

15. Quinn EM, Kearney DE, Kelly J, et al. Temporal artery biopsy is not required in all cases of suspected giant cell arteritis. Ann Vasc Surg. 2012;26(5):649-54.

16. Garcia-Garcia J, Ayo-Martin O, Argandona-Palacios L, et al. Vertebral artery halo sign in patients with stroke: a key clue for the prompt diagnosis of giant cell arteritis. Stroke. 2011;42(11): 3287-90.

17. Wiszniewska M, Devuyst G, Bogousslavsky J. Giant cell arteritis as a cause of first-ever stroke. Cerebrovasc Dis. 2007;24(2-3): 226-30. 